\title{
Enhancing pandemic resilience: planning and institutional resilience, a learning model for Indonesia
}

\author{
Marnani. Christine $\mathrm{S}^{1^{*}}$,Hartanto $^{2}$, and Sobar Sutisna ${ }^{1}$ \\ ${ }^{1}$ Disaster Management Study Program, National Security Faculty, Republic of Indonesia Defense University. IPSC Sentul Area, \\ Bogor, West Java, Indonesia \\ ${ }^{2}$ Department of International Relations, Respati Yogyakarta University
}

\begin{abstract}
The COVID-19 pandemic is one of the most expensive and destructive threats facing millions of people around the world. Policymakers generally have a linear worldview and can help the economy and society to track epidemic shocks and crises by pulling the right levers. For example, in recent years, Ebola, MERS, Zika, and SARS have demonstrated a limited willingness to respond promptly and responsibly. The pandemic emphasizes the need to be more proactive. Yet, existing epidemic response frameworks are either disease-specific, category-specific, or non-specific, with no comprehensive framework encompassing all components of institutional resilience. In addition, the COVID-19 pandemic requires a holistic approach to meet the challenges. In this study, the goal of resilience to the outbreak of the epidemic is to develop an overall framework by identifying institutional departments to strengthen resilience planning. The framework can guide evidence-based decision-making and prioritization. This article encapsulates a framework that focuses on the interactions between processes and their system characteristics, which leads to an overemphasis on a limited number of features, significantly enhancing the flexibility of the organization
\end{abstract}

\section{Introduction}

Environmental emergencies were rarely prioritized in catastrophe planning, development sustainability, or business continuity. Anthropogenic, climate-changerelated, or technological accidents involving ecological or biological systems have taken a significant amount of the risk-space we live in recent years. Health catastrophes of the magnitude that the COVID-19 pandemic has brought to the world's attention necessitate a rigorous study of the processes and dangers of such environmental and health outbreaks. This is crucial to comprehending the emerging issues surrounding low-frequency high-intensity disasters such as pandemics, tsunamis, floods, liquefaction, water contamination emergencies, landslide, or volcanic eruptions causing death and food shortages in specific areas.

The topic was written in response to the necessity of learning from the COVID-19 health catastrophe, which turned into a massive global calamity. The lessons learned from this pandemic calamity are helpful not only for health and social systems but also for the economy and business sectors, environmental protection, and the pursuit of sustainable development goals. The study summarises and recommends lessons learned from crossdomain assessments of worldwide virus outbreaks, and it will be beneficial in creating disease-resistant populations. Scholars in humanities, management, and public policy intend to use this study as a knowledge support system and reading reference, especially for Indonesian scholars.

\section{Pandemic: An Overview}

Pandemics have shaped human history $[1,2,3]$, but the name "pandemic" is new. Modern cultures have the dubious fortune of experiencing near-simultaneous epidemics over the world as a result of several centuries of relentlessly growing globalization. An increase in the number of developing diseases over the last two decades has fueled fears of a new devastating pandemic on the horizon. Nonetheless, much of the globe was caught off guard when the World Health Organisation (WHO) proclaimed the developing coronavirus SARS-CoV-2 a pandemic on March 11, 2020.

Since the first coronavirus disease of 2019 (COVID19) was discovered in the Chinese city of Wuhan in December 2019, the globe has been facing a crisis on numerous fronts, including public health, social welfare, and economic development. This global problem does not appear to be slowing down, and the disease's effects are posing new challenges to governments worldwide. According to a general understanding of similar diseases in the past, the evolution, spread, and fatality of such

\footnotetext{
* Corresponding Author: christinemarnani@yahoo.com
} 
epidemics are dependent on both natural and human variables. Many investigations have been undertaken to determine the disease's complex link with natural and anthropogenic variables.

For example, Zhou revealed in a significant study that individuals with pre-existing medical conditions are more susceptible to COVID-19 [4]. People with severe disease conditions are more vulnerable to high health repercussions [5]. These elements, particularly in developing nations where health infrastructure is inadequate and socioeconomic conditions are insufficient to deal with the pandemic's effects, play a critical influence in determining fatality rates. According to a study of African countries' healthcare systems, the COVID-19 pandemic poses a significant threat to the continent on numerous levels [6].

Not only is the continent ill-prepared to combat such a sickness, but it has also been shown to be vulnerable to the effects of such a large-scale outbreak. Similarly, several scholars have looked into the pandemic's economic repercussions. For example, Zhang discovered that the COVID-19 has proven to be one of the most important contributors to increasing global financial market volatility [7]. The study of several micro and macro variables in various disciplines found that significant efforts and resources will be required to limit the amount of harm, particularly in developing countries with higher population density [8].

$\mathrm{Qu}$ has suggested that environmental variables play a role in the pandemic spread. Although several studies have been conducted to explain the complex interaction between environmental factors and COVID-19, there is still a lack of consensus among researchers and scientists on the subject [9]. For example, $\mathrm{Wu}$ found that a rise in temperature and humidity could help to restrict the COVID-19 pandemic to some extent, based on data from more than 150 nations [10].

However, Zhu and Xie found that an increase of one degree Celsius in the mean temperature was positively connected with roughly $5 \%$ of the infected cases after studying the growth of instances of more than 100 in China. They concluded that there was no compelling evidence of COVID-19 confirmed cases associated with possible warming scenarios [11]. However, earlier research has shown that the wide range of diseases caused by the 'severe acute respiratory syndrome (SARS) virus family are linked to environmental factors such as pollution, temperature, relative humidity, and various other climatic parameters $[12,13,14]$. Given the above debates, these studies call decision-makers' attention to the need to increase the population's capacity, particularly in rural areas. The density of educated people is also a factor in pandemic vulnerability. This will help policymakers create more effective risk measuring, readiness, response, and mitigation programs.

\section{Pandemic's Effects}

Even though pandemics are rare occurrences, they have a significant impact on the world's shaping. The consequences necessitate well-thought-out contingency plans to prevent disease spread and death. While containment methods are implemented in stages, and medical therapies take time, the pandemic has significant economic, financial, and social consequences.

In many ways, the COVID-19 pandemic is similar to earlier pandemics, according to an analysis of their economic implications. Except for the Spanish flu, which spread through military networks, trade and travel by land, air, or sea propagated other pandemics. As in the case of swine flu, the greater the extent of global trade, the faster the virus spreads. Because of the rising demand for healthcare items, there has been a surge in healthcare service businesses. Higher pricing and strains on healthcare spending have resulted because of this. Due to the lack of products, prices were higher during the Ebola outbreak. Some pandemics, such as the Black Death and the Spanish Flu (both had a high death rate), resulting in a labor shortage and increased salaries.

Work has been disrupted, schools and companies have closed, and as a result, productivity has decreased. Hotels and the entertainment business have suffered as a result of the downturn in the service sector. In more recent pandemics, there had been a drop in tourism and travel earnings (2000 onwards). The effect on productivity, on the other hand, is debatable. It was discovered that the Spanish flu resulted in higher capital per worker and thus higher productivity. However, another study found low long-term productivity due to lost skills (due to unemployment) and a negative impact on the productivity of children born to moms who were pregnant during the epidemic. In the aftermath of Ebola, there was a drop in productivity and participation in the labor force.

GDP, investment, and consumer confidence have all been negatively impacted. For countries that rely heavily on tourism, the impact on GDP was larger. Pandemics that occurred before the twenty-first century had little effect on trade, especially in nations like Guinea, Liberia, and Sierra Leone, which had a small share of the global economy. The news of the pandemic has a significant impact on market sentiment and fear.

Though it may appear that societal implications are boosting short-term stress levels and posing issues in terms of women's abuse, child labor, and domestic violence, these can have far-reaching consequences that have the potential to worsen conditions in the future. Because there are significant interdependencies between social features and economic, political, and financial aspects, it is critical to limit these negative impacts on the economy's bright future.

In terms of international coverage and transmission speed, COVID-19 stands out. "Preliminary medical investigations suggest that COVID-19 is less lethal than SARS but more transmissible [15]. Because the world is now much more integrated in terms of trade and travel, it has absorbed far more countries than earlier pandemics. Because previous pandemics spread primarily through trade and travel routes, it is obvious that growing economic integration has made countries more vulnerable to the severe economic effects of such pandemics.

The list of the ten countries severely afflicted by COVID-19 is nearly equal to the list of the world's ten largest economies (Iran and India are the exceptions) [16]. 
The United States, China, Japan, Germany, the United Kingdom, France, and Italy are among the top 10 countries impacted by the disease. Just the United States, China, Japan, Germany, the United Kingdom, France, and Italy account for $60 \%$ of global supply and demand (GDP), 65 percent of global manufacturing, and $41 \%$ global manufacturing exports". These factors indicate that a modern-day pandemic such as COVID-19 will have far more significant economic implications for the entire planet than any other pandemic previously witnessed.

\section{Institutional Resilience}

The pandemic of COVID-19 is among the most expensive and devastating threats to millions of people worldwide. On June 4, 2020, the highest death rate of 315.54 per million was being faced by the United States of America (USA), whereas a 190.93 death rate per million people for Canada and a lower death rate of 0.027 per million for Indonesia. The first confirmed case of COVID-19 virus infection was in Jakarta. She had been in contact with a Japanese citizen who tested positive in Malaysia on February 27, 2020. Then onwards, Indonesia is facing an exponential increase in the number of COVID-19 patients with symptomatic and asymptomatic symptoms.

Infectious illness data-driven frameworks are increasingly being utilized to provide real-time or nearreal-time situational awareness during disease outbreaks. Despite the inherent constraints of predicting the size and magnitude of complex processes, mathematical and computational models have been employed to anticipate epidemic size and scale. This aids in calculating the danger of case importation around the world and the risk of disease outbreaks that go unchecked [17]. Despite differing viewpoints on using modeling tools in epidemiological studies, many studies have been conducted in recent years to evaluate infection containment and mitigation tactics and develop pandemic preparation warning systems. In most cases, model-based epidemic scenarios concentrate on the magnitude and length of the epidemic or pandemic [18].

In addition, the modeling community is presently evaluating resilience, mitigation, and containment policies by lowering the attack rate per hundreds, thousands, or tens of thousands of persons in the population. The evaluation of epidemic risks and consequences is a multifaceted challenge that must incorporate societal issues such as infrastructure and service interruption, lost output, inflated prices, crisisinduced budget deficits, and poverty [19]. As a result, it's critical to expand the framework-based approach to epidemic analysis. This framework will include measurements that can be used to assess systemic and institutional resilience, such as the overall system's response to disruptions, the implications of those disruptions, the outcome of mitigation, and the recovery and retention of system functionalities [20].

COVID-19 outbreaks have a multi-cascade effect, affecting many industries and perhaps resulting in worldwide institutional crises and poor economic development. Understanding these areas is vital for human survival, and their resilience will serve as important indicators. Institutional performance is partly dependent on robust growth, but mostly on resilience: the ability to bounce back, develop workflows that absorb losses and deficiencies, be aware of and participate in changing conditions and feedback, and deal methodically with the pattern of problems that will inevitably arise along the way [21].

Although there has been a lot of research on evaluating the resistance of intricate processes to unfavorable events, its integration into the analysis technique for epidemic hazards is still mainly unexplored. Most crucially, institutional resilience must include the temporal horizon or the recovery and stability of functionality in the case of an epidemic $[22,23,24]$. As a result, identifying and managing institutional resilience to epidemics requires assessing the temporal profile of how essential functions of the system are kept or managed to recover in response to pandemic events.

Mitigation, preparedness, response, and recovery are well-known disaster phases; however, they don't work well as frameworks for Institutional Resilience. Social media impacts the intricacies of national and international institutions such as banking, trade, and communication rather than communities [25]. The different activities of epidemic response, the links between them, and potential avenues for an impact have been developed for an epidemic response to this review to determine the different activities of epidemic response, the links between them, and potential avenues for an impact.

COVID-19 pandemics, which differ from epidemics in that they have more defined policy aims, pose similar issues in terms of establishing understanding frameworks. Many frameworks, for example, were created to develop appropriate interventions, set priorities during the implementation phase, or assess specific aspects of performance in many circumstances. A comprehensive action encompassing all approaches to examine all stages of a life cycle intervention was not taken. As a result, an improved framework consisting of preparedness, coping mechanisms, adaptation, awareness, and transformation has been identified as a means of improving institutional resilience.

\subsection{Preparedness}

Five EU member states have been affected by Middle East Respiratory Syndrome (MERS) and poliomyelitis, including the UK, Greece, Spain, Poland, and Cyprus. Policies and tactics exist in all nations, and valuable lessons have been learned and institutionalized as a result of previous public health catastrophes such as Ebola (2014-2016), the H1N1 pandemic (2009), and SARS (2003). [26, 27, 28]. There were also significant lasting benefits from large meetings like the Olympics, held in Greece in 2004, and the United Kingdom in 2012. However, new technology, knowledge, and experience gained from these events are subject to political and policy priorities shifting away from securing effective core public health capacities in some participating countries [29]. There was no preparedness for COVID-19, but the 
lessons acquired from earlier outbreaks have presented institutions with strategies to improve their preparedness.

There are crucial measures to ensuring that European public health preparedness programs are ready to conduct evaluations, document outcomes, and communicate them broadly, such as frequent training of all necessary staff personnel [30]. A similar methodology can be used in poorer countries to improve institutional preparation. More catastrophe preparedness planning is needed, and institutional processes must be integrated. Multi-scalar integration and governance structures that enable cohesive collaboration and cooperation within the system are part of this readiness for national and local institutional systems with shorter supply chains.

\subsection{Coping Mechanism}

Resilience is more challenging and necessitates acts that may appear contradictory to logic at times. A literature review indicates three critical measures they may take to strengthen the resilience of their personnel, teams, and institutions [31]. The ability to manage with the consequences of economic losses, no employment, job recession, and insecurity in the demand and supply chain emphasizes the importance of coping needs in improving the institutional resilience of businesses and governmental structures [32]. It needs to Adopt a systemic approach to health policy to create flexible, resilient, sustainable, and equitable resilient systems.

\subsection{Adaptation}

Over the last 30 years, the incidence of epidemics has led to increasing adaptation measures for dealing with them. Outbreaks of such diseases and pandemics impair transportation, food, health, tourism, education, and services, resulting in lost output, inflated prices, a crisisinduced budget deficit, and poverty [33]. The supply chain suffers the greatest, affecting all areas of society. A systemic shift is also being witnessed around the globe, both in developed and developing countries, from spending on luxury items to spending on necessities such as life-saving pharmaceuticals, which is improving resilience structures.

\subsection{Awareness and Transformation}

Increased awareness is a necessary component of improving institutional resilience. During the Ebola outbreak, promoting awareness served as a strategic means of overcoming the crisis. It aided in improving Liberia's health system's resilience to the Ebola epidemic [34]. Furthermore, improving social media networks to ensure their viability would assist in raising awareness. The importance of knowledge sharing will improve epidemic response. The sharing of value is needed as a means of disseminating best practices and lessons learned as well as fostering solidarity from the communities to elevate assistance. Science must be measured in terms of various types of learning. Small farmers, indigenous peoples, women, and young people must actively raise their voices.

The national-local link is critical for guaranteeing policy and implementation continuity. The incorporation of readiness, scoping, adaptation, and awareness into the system results in modifying several concepts, designs, and analyses [35]. Transformation in all kinds of structural reformation and prioritizing strengthen the establishment of a resilient foundation. These are country-specific because each has a unique collection of resources and capacity to deal with epidemics.

\section{Institutional Planning}

With rising concerns about environmental challenges/climate change and developing disease scenarios, it is critical to consider the creation of sustainable production systems. The need for planning in an organization for any business or activity is expanding to society's constant logical progress today [36]. When it comes to corporate activity planning, the function of management tools is crucial. Effective management tool use is a possible actor for long-term planning and production practices [37]. This section discusses some significant instruments that are useful when it comes to institutional planning.

\subsection{Evaluation of The Life Cycle}

It is a system for examining the environmental consequences of all processes connected with all stages of product, service, and process development. This system is a critical tool for industries, businesses, and policymakers to properly strategize their decisions and operations. It employs a cradle-to-grave method that provides a full review of the entire organizational structure, including up and down chain parts and third-party elements involved in the system. Doing so decreases the impact of external demand on organizational performance and action approaches, boosting organizational resilience.

\subsection{Cost-Benefit Analysis}

It is a system for assessing the environmental impacts of all processes associated with all stages of product, service, and process development. The Cost-Benefit Analysis is an essential tool for industries, businesses, and regulators to incorrectly strategize their decisions and operations. It uses a cradle-to-grave approach to give a comprehensive examination of the complete organizational structure, encompassing up and down chain portions and third-party elements involved in the system. It reduces the impact of external demand on organizational performance and action approaches, hence increasing organizational resilience.

\subsection{On-Site and Off-Site Planning}

Emergencies necessitate urgent action, and adequate preparation is required to carry out these measures. 
Emergency preparedness is needed for any organization to respond to emergencies both on and off-site [37]. Onsite planning is specific to dealing with the consequences of any emergency event that disrupts internal operations, structures, or workforce. The emergency response needs of the areas surrounding the organization are addressed via site planning. Before generating operational plans, onsite and off-site studies are valuable for prioritizing emergency response planning.

\subsection{Exercises in Impact Dependence}

Businesses, organizations, and institutions rely on biodiversity (through ecosystems and associated ecosystem services) to run their operations and activities smoothly. Any disturbance in the critical ecosystems and related services on which organizations rely will significantly impact their (structural and functional) operations. Conducting impact dependency exercises assists businesses in future-proofing their operations by [38]: ensuring the future supply of vital resources, improving resource efficiency, securing future operating licenses, should plan better business decisions and activities. It also aids in spotting company risks and identifying new opportunities.

\subsection{Assessing Vulnerability}

Environmental issues, such as natural catastrophes or epidemics, impede organizational processes by disrupting services and causing infrastructure damage. Vulnerability assessment is a tool that aids in finding hotspots or zones/areas that are most vulnerable to risks. Conducting a vulnerability assessment helps identify the required adaptive responses - whether the organization's structure and processes have the necessary coping and adaptive capability to take on or move forward with specific activities [39].

\subsection{Continuity Preparation}

Business continuity planning is a proactive strategy to understand an organization's or institution's weaknesses and risks when a crisis arises. The fundamental strategy is to maintain critical functions operating and eventually recover with minimal downtime, preventing systems from failing [39]. Effective planning enables organizations to respond quickly and efficiently in the face of unexpected events, building risk management systems to avoid disruptions in operations. A business continuity plan is built on three major components: Resilience: Considering many adversities during operation planning and design improves organizational resilience for multiple risks. Recovery: During a crisis, focusing only on operations that include critical/essential services should be preferred, followed by establishing a recovery time aim for the rest of the functions. Contingency Plan: Ensures that proper procedures are in place for various internal work plans and value chain points [40].

\section{Conclusion}

The current coronavirus or COVID-19 pandemic has taught many important lessons, and many people are now discussing how to deal with crisis inside crisis. Incidences of crisis during a crisis are not new; instead, they have a long history. However, it is critical to consider what lessons we have gained from previous situations and if they have been incorporated into policies and management practices. As a result, the emerging field of resilience assessment and administration, as well as its implementation, could assess cross-domain alternatives to identify a policy design that improves the system's ability to (1) plan adverse events, (2) absorb stress, (3) recover, and (4) prevent and prepare for future stressors by appropriately adapting them. System planning should be inclusive, considering the entire value chain and evaluating life cycle components into planning procedures. To summarise, the paradigm we described may help balance reduced risk with disruption to important activities linked with public health interventions to optimize policy outcomes.

\section{References}

1. WH. McNeill, Plagues and peoples (Garden City, New York, 1976)

2. JM. Diamond, Guns, Germs, and Steel: The Fates of Human Societies (W.W. Norton \& Cole, New York, 1999)

3. A. Crosby, The Columbian exchange: biological and cultural consequences of 1492 (Greenwood, Westport, 1972).

4. F. Zhou, T. Yu, R. Du, G. Fan, Y. Liu, Z Liu, J. Xiang, Y. Wang, B. Song, X. Gu. L 395(10229): 1054-1062 (2020)

5. L. Fang, G. Karakiulakis, M. Roth. LRM 8 (4):e21(2020)

6. JN. Nkengasong, W Mankoula (2020). L 395,:841842 (2020)

7. D. Zhang, M. Hu, Q. Ji, FRL:101528 (2020)

8. WJ. McKibbin, R. Fernando, The global macroeconomic impacts of COVID-19: seven scenarios (March 2, 2020). CAMA Working Paper No. 19/2020. (2020)

9. G. Qu, X. Li, L. Hu, G. Jiang. EST 54 (7), :37303732 (2020).

10. Y. Wu, W. Jing, J. Liu, Q. Ma, J. Yuan, Y. Wang, M. Du, M. Liu, STE 729,:139051 (2020).

11. Y. Zhu, J. Xie. STE 724,:138201 (2020).

12. J. Bao, Z. Wang, C. Yu, X. Li. BMC Public Health 16:375 (2016).

13. Y. Cui, Z-F. Zhang, J. Froines, J. Zhao, H. Wang, SZ. Yu, R. Detels. EH 2:15 (2003).

14. K. Lin, DY-T. Fong, B. Zhu, Karlberg. 134,:223-230 (2006). 
15. R. Baldwin, di Mauro BW, Economics in the time of COVID-19. (CEPR Press, Washington, DC, 2020).

16. Q. Zhang, H. Deng, C. Zhang. The value of early response by surrounding areas of epidemic center Hubei during COVID-2019 outbreak in China: a quasi-experiment analysis. Available at SSRN 3548372 (2020).

17. E. Massaro, A. Ganin, N. Perra, I. Linkov, A. Vespignani, SR 8(1):1-9 (2018).

18. AA. Ganin, E. Massaro, A. Gutfraind, N. Steen, JM. Keisler, Kott A et al. SR 6(1):1-12 (2016)

19. G. O'Brien, UK emergency preparedness: a holistic response? DPM 17(2):232-243 (2008).

20. E. Massaro, A. Ganin, N. Perra, I. Linkov, A. Vespignani, SR 8(1):1-9 (2018).

21. J. Wang, Z. Wang, Strengths, weaknesses, opportunities and threats (SWOT) analysis of China's prevention and control strategy for the COVID-19 epidemic. IJERPH 17(7):2235 (2020).

22. J. Romaní, E. Baselga, O. Mitjà, N. Riera-Martí, P. Garbayo, Vicente A et al, AD 111(5):426-429 (2020).

23. CA. Bevc, MC. Simon, TA. Montoya, JA. Horney, PHR 129:35-41 (2014)

24. DJ. Dausey, M. Moore, BMC Res Notes 7(1):474 (2014)

25. J. Kinsman, J. Angrén, F. Elgh, M. Furberg, PA. Mosquera, L. Otero-García, R. Snacken, T.
Derrough, PC. Santisteve, M. Ciotti, S. Tsolova, BMC Health Serv Res 18(1):528 (2018)

26. B. Aguirre, RR. Dynes, J. Kendra, R. Connell, JHSEM 2(2) (2005)

27. MA. Barton, M. Christianson, CG. Myers, K. Sutcliffe. Resilience in action: leading for resilience in response to COVID-19. BMJ Leader, pp leader(2020)

28. A. Warsame, K. Blanchet, F. Checchi, BMJ Glob Health 5(1):e002109 (2020)

29. EJ. Ling, E. Larson, RJ. Macauley, Y. Kodl, B. VanDeBogert, S. Baawo, ME. Kruk, HPP 32, iii40iii47 (2017)

30. AA. Ganin, E. Massaro, A. Gutfraind, N. Steen, JM. Keisler, Kott A et al. SR 6(1):1-12 (2016)

31. UNEP, Benefits of life cycle approaches. Life Cycle Initiative (UNEP, New York, 2020)

32. LfN I (2012) Why follow the natural capital roadmap? Leaders for Nature. http://leadersfornature.in/get-started. [Accessed June 3, 2020]

33. Bindal, Sonal, Pritha Acharya, Anil Kumar Gupta, and Jugal Kishore. "Enhancing Epidemic Resilience: Planning and Institutional Resilience." In Integrated Risk of Pandemic: COVID-19 Impacts, Resilience, and Recommendations, 463-480. (Springer, Singapore, 2020) 